\title{
Omega-3 index and smoking in patients with acute ST-elevation myocardial infarction taking statins: a case-control study in Korea
}

Young Joo Kim ${ }^{1 \dagger}$, Dong Wook Jeong ${ }^{2 \dagger}$, Jeong Gyu Lee ${ }^{1 \dagger}$, Han Cheol Lee ${ }^{3}$, Sang Yeoup Lee ${ }^{2,4,6^{*}}$, Yun Jin Kim', Yu Hyeon $\mathrm{Yi}^{1}$, Yong Soon Park ${ }^{5}$, Young Hye Cho ${ }^{2}, \mathrm{Mi}$ Jin Bae ${ }^{1}$ and Eun Jung Choi ${ }^{2}$

\begin{abstract}
Background: n-3 fatty acids and lifestyle also are closely related to risk of CVD. Most Koreans have higher fish consumption than people of Western populations. However, little is known about the recommended value of omega-3 index in Korean patients with acute ST-elevation myocardial infarction (STEMI) taking statins. Here, we tested the hypothesis that lower omega-3 fatty acids and/or smoking are associated with acute STEMI, even though patients with dyslipidemia who were taking statins and who attained their LDL-C goals.
\end{abstract}

Methods: We conducted a case-control study in which omega-3 fatty acids and lifestyle factors were determined in 24 consecutive Korean patients taking statins with angiographically confirmed acute STEMI and 68 healthy controls without acute STEMI. The omega-3 index was calculated by the sum of eicosapentaenoic acid and docosahexaenoic acid in erythrocyte membranes. Multivariable adjusted regression analysis was used to assess independent associations between acute STEMI, omega-3 index, and lifestyle factors after adjusting for age, sex, and body mass index (BMI).

Results: The mean age of total subjects was 59.9 years, and $57.6 \%$ of the subjects were male. The omega-3 index was significantly lower in cases (8.83\%) than controls (11.13\%; $P<0.001)$; however, total trans-fatty acids were not different between the two groups. The omega-3 index was inversely associated with odds for being a case (OR 0.16 (95\% Cl 0.03-1.14); $\mathrm{P}=0.047$ ), while smoking was positively associated with odds for being a case (OR 6.67 (95\% Cl 1.77-25.23); $\mathrm{P}=0.005$ ) after adjusting for all confounding variables.

Conclusion: This study shows that relative to controls, acute STEMI cases are more likely to be smokers and to have a lower omega-3 index, even though the cases were taking statins. An omega-3 index of at least 11\% and abstinence from smoking are associated with cardioprotection for Koreans.

Keywords: Omega-3 index, Smoking, Myocardial infarction, Fatty acid, Fish oil

\section{Background}

Cardiovascular disease (CVD) is one of the most common causes of mortality and morbidity across the globe [1]. Studies that have focused on the prevention of coronary heart disease (CHD) have reported that lifestyle modifications such as smoking cessation, adequate alcohol consumption, exercise, stress reduction, weight

\footnotetext{
* Correspondence: saylee@pnu.edu

+ Contributed equally

${ }^{2}$ Family Medicine Clinic and Research Institute of Convergence of Biomedical Science and Technology, Pusan National University Yangsan Hospital,

Yangsan, South Korea

Full list of author information is available at the end of the article
}

control, and diet are preventable factors of CVD [2-5]. Dietary $n-3$ polyunsaturated fatty acids (PUFA) such as eicosapentaenoic acid (EPA) and docosahexaenoic acid (DHA), which are found in fish meat and fish oil, have also been shown to protect against CVD during the past three decades [6]. Since the first observation of Greenland Eskimos in 1970, epidemiologic studies have suggested that $n-3$ PUFA may play a role in the prevention of CVD [7]. Several large-scale intervention studies have reported that n-3 PUFA from fish or fish-oil supplements reduces the rates of all-cause mortality, cardiac and sudden death, and possibly stroke [8]. In addition, 
$n-3$ fatty acids are considered to have several beneficial effects, including reduction of inflammation [9], prevention of blood clotting [10], decrease in triglycerides [11], and lowering of blood pressure [12].

The sum of EPA + DHA in erythrocyte (RBC) membrane fatty acids is a good reflection of systemic n-3 PUFA status and is called the omega- 3 index, which is expressed as a percent of total identified fatty acids [13]. Previous studies have shown that Asians with higher fish consumption had a greater omega- 3 index, and furthermore, they had a higher omega-3 index than the recommended value of $8 \%$ or greater to prevent CHD for Western people. The omega-3 index in healthy Koreans and Japanese is about $11-12$ and $7-11 \%$, respectively, while it is around $4 \%$ in people from Western populations [13-15]. Nevertheless, CVD is becoming more prevalent in Korea and is now a leading cause of death [16], although the prevalence of CVD in Korea remains lower than that in the US and other Western populations. A study of more than 40,000 middle-aged Japanese people revealed that a higher fish intake is associated with a substantially reduced risk of CHD compared to the recommended amount of fish intake in Western countries [17].

A recent meta-analysis of 3-hydroxy-3-methylglutaryl coenzyme A reductase inhibitors (statins) in primary prevention showed that the lipid profile was improved in all 14 trials of about 34,000 people who had no evidence of existing CVD, but the benefits of statins for the primary prevention of CVD may differ according to cardiovascular risk [18]. Recently, several studies confirmed that prescription omega-3 fatty acids administered in statin-treated patients with hypertriglyceridemia was superior to statins alone in improving lipid parameters [19-21]. However, it remains unclear whether omega- 3 index is different between controls and patients with recent myocardial infarction (MI) taking statins in Koreans, who consume more fish than Western populations. The purpose of the present study was to compare fatty acid composition of RBCs, the omega-3 index, and lifestyle behaviors between controls and patients with acute ST-elevation MI (STEMI) who attained their LDL-C goals with statins.

\section{Materials and methods}

\section{Study design and subjects}

This was a cross-sectional, case-control study. The study protocol was approved by the Institutional Review Board of Pusan National University Hospital, and informed written consent was obtained from all subjects before participating.

Subjects were divided into 2 subgroups: a case group of 24 patients with first acute MI, who were consecutively recruited from the Coronary Care Unit at Pusan National University Hospital (Busan, Korea) and a control group of 68 apparently healthy persons with no history or clinical evidence of CVD, hypertension, or diabetes mellitus as well as acute MI, who were not taking statins and recruited from the Health Promotion Center during the course of annual health check-up visits.

Emergency coronary angiography was performed using the percutaneous femoral approach within the first $12 \mathrm{~h}$ after the onset of typical ischaemic-type chest pain. Acute STEMI was defined according to consensus documents of American College of Cardiology and European Society of Cardiology as typical ischaemic-type chest pain, typical rise and fall of biochemical markers (troponin or CK-MB), and electrocardiogram showed new ST segment elevation or pathologic $\mathrm{Q}$ waves. The number and severity of coronary artery stenosis were documented through coronary angiography. All patients were already taking statins due to dyslipidemia with/without hypertension or type 2 diabetes mellitus. Exclusion criteria included abnormal liver or renal function (ie, serum aminotransferase activity $>40$ $\mathrm{IU} / \mathrm{L}$ and serum creatinine concentrations $>1.2 \mathrm{mg} / \mathrm{dL}$ ); cancer (diagnosed clinically or by anamnesis); extreme weight loss or gain over the previous 6 months; thyroid or pituitary disease; infection determined by medical questionnaire and complete blood count; and connective tissue disease.

Clinical, sociodemographic, lifestyle, and medication information such as oral hypoglycemic agents, insulin, lipid-lowering agents, and anti-hypertensive or estrogen agents were obtained from all study subjects. Subjects taking a supplement containing $\mathrm{n}-3$ fatty acids were excluded.

\section{Measurements}

Height and body weight of subjects were measured using a digital scale, and the body mass index (BMI) was calculated as weight $(\mathrm{kg}) /$ height $\left(\mathrm{m}^{2}\right)$. Resting blood pressure was measured using an automatic sphygmomanometer (BP203RV-II; Nippon Colin, Komaki, Japan) after > 10 $\mathrm{min}$ at rest in a sitting position.

Peripheral blood was drawn from patients on day 1 (at the time of admission) after the onset of infarction and controls after an overnight fast of at least $8 \mathrm{~h}$ for measurements of serum total cholesterol, triglycerides, high density lipoprotein (HDL)-cholesterol, low density lipoprotein (LDL)-cholesterol, and glucose. All biochemical analyses were carried out within $2 \mathrm{hr}$ of blood sampling using an autoanalyzer (model 7600-110; Hitachi Corp., Tokyo, Japan) and commercially available kits. RBCs were used for fatty acid analysis. Boron trifluoride methanol-benzene (BI252; Sigma-Aldrich, BI252; Sigma-Aldrich, St. Louis, MO, USA) was added to RBCs, and samples were methylated for $10 \mathrm{~min}$ at $100^{\circ} \mathrm{C}$. Fatty acid methyl esters were analyzed by gas chromatography (Shimadzu 2010 AF; Shimadzu Scientific Instrument, Tokyo, Japan) with a $100 \mathrm{~mm} \times 0.25 \mathrm{~mm}$ SP2560 capillary column (Supelco; 
Bellefonte, PA, USA). Standard gas liquid chromatography (GLC-727; Nu-Check Prep, Elysian, MN, USA) was used for identifying fatty acids and correcting inter-assay variation. In the standard 18: 1t peak was the mixture of 18 : 1n-12t, C18: $1 n-9 \mathrm{t}$, and 18: $1 n-7 \mathrm{t}$, while the 18: $2 n$-6t peak contained 18: $2 n-6 \mathrm{tt}, 18: 2 \mathrm{n}-6 \mathrm{tc}$, and 18:2n-6ct. Fatty acids for which no standards were available are reported as unidentified fatty acids. When the percentage of fatty acid was less than 05 , it is recorded as a trace. The omega- 3 index was calculated as the sum of EPA and DHA in RBCs and expressed as percentage of total fatty acids in the RBC membrane [22]. The quality-control sample was composed of pooled RBCs, and the coefficient of variation was $6.2 \%$.

The usual dietary intakes were assessed using a semiquantitative food frequency questionnaire (FFQ). A trained dietitian interviewed the participants regarding the average frequency of consumption and portion size of each food weekly. FFQ was designed to assess the habitual diet during the previous seven days, the daily intakes of food groups, energy, and nutrients were computed using an analysis program, and weekly total fish intakes were obtained from all subjects. Alcohol consumption and smoking status were assessed using a self-reported questionnaire. Data on alcohol intake and smoking habits were obtained by interview. Subjects were divided into two groups by the amount of alcohol consumption: non-drinker, 0-180 g/week; and drinker, > $180 \mathrm{~g} /$ week. Based on WHO guidelines, smoking status was divided into three categories: current smoker, ex-smoker, and non-smoker.

\section{Statistical analysis}

The D'Agostino-Pearson test was used to test the normality of all continuous variables. Results are shown as mean and s.d. because variables were all normally distributed. The two sample $t$-test and chi-square test were applied for continuous and categorical variables, respectively. Correlation between variables was tested by partial correlation coefficients after adjusting for age and sex. Multivariable adjusted regression analysis was used to assess independent associations between acute STEMI, quartiles of the omega-3 index, and lifestyle factors after adjusting for age, sex, and BMI. Statistical analysis was obtained using the Statistical Package for Social Science 13.0 for Windows (SPSS, Inc., Chicago, USA). All $\mathrm{p}$ values were two-tailed, and a $\mathrm{p}$ value of $<0.05$ was considered statistically significant.

\section{Results}

\section{General characteristics of study subjects}

The basal characteristics of subjects are shown in Table 1. Among all acute STEMI patients having multi-vessel coronary artery disease, $12(50.0 \%)$ had one-vessel disease, 5 (20.8\%) had two-vessel disease, and 7 (29.2\%) had three-
Table 1 Sociodemographic and clinical characteristics of study subjects

\begin{tabular}{lccc}
\hline & Case $(\mathbf{n}=\mathbf{2 4})$ & Control $(\mathbf{n}=\mathbf{6 8})$ & $\mathbf{p}$ value \\
\hline Age (years) & $61.6 \pm 11.6$ & $59.3 \pm 8.1$ & 0.370 \\
Men (\%) & $17(70.8)$ & $36(52.9)$ & 0.127 \\
Body mass index $\left(\mathrm{kg} / \mathrm{m}^{2}\right)$ & $23.5 \pm 2.8$ & $24.0 \pm 2.5$ & 0.405 \\
Total cholesterol $(\mathrm{mg} / \mathrm{dl})$ & $159.0 \pm 30.0$ & $201.1 \pm 38.8$ & $<0.001$ \\
Triglyceride $(\mathrm{mg} / \mathrm{dl})$ & $123.3 \pm 73.9$ & $128.7 \pm 62.8$ & 0.731 \\
HDL-cholesterol $(\mathrm{mg} / \mathrm{dl})$ & $42.1 \pm 10.8$ & $52.9 \pm 10.7$ & $<0.001$ \\
LDL-cholesterol $(\mathrm{mg} / \mathrm{dl})$ & $81.4 \pm 24.7$ & $119.8 \pm 34.7$ & $<0.001$ \\
Systolic BP $(\mathrm{mm} / \mathrm{Hg})$ & $111.9 \pm 18.7$ & $124.3 \pm 14.1$ & 0.001 \\
Diastolic BP $(\mathrm{mm} / \mathrm{Hg})$ & $70.5 \pm 13.0$ & $76.0 \pm 8.6$ & 0.065 \\
hs-CRP (mg/dl) & $2.66 \pm 3.50$ & $0.13 \pm 0.19$ & 0.002 \\
Co-morbidity & & & \\
$\quad 10(41.7)$ & $0(0.0)$ & $<0.001$ \\
$\quad$ Hypertension & $12(50.0)$ & $0(0.0)$ & $<0.001$ \\
$\quad$ Diabetes mellitus & $24(100.0)$ & $0(0.0)$ & $<0.001$ \\
$\quad$ Dyslipidemia & $2.1 \pm 2.1$ & $3.1 \pm 2.1$ & 0.059 \\
Fish intake (servings/week) & $10(41.7)$ & $21(30.9)$ & 0.337 \\
Alcohol drinker (\%) & $11(45.8)$ & $10(14.7)$ & 0.002 \\
Current smoker (\%) &
\end{tabular}

Data are expressed as means \pm SD or number (\%).

$\mathrm{HDL}$, high density lipoprotein; $\mathrm{LDL}$, low density lipoprotein; BP, blood pressure; hs-CRP, high sensitivity C-reactive protein.

$\mathrm{p}$ value by two sample $t$-test or chi-square test

vessel disease. The mean age of total subjects was $59.9 \pm$ 9.1 years, and $57.6 \%$ of the subjects were male. Looking at the case-control comparison, the case group had significantly lower levels of total cholesterol, LDL-cholesterol, HDL-cholesterol, and systolic blood pressure and higher levels of high sensitivity $\mathrm{C}$-reactive protein than did the control group. However, there was no significant difference in age, gender distribution, BMI, triglyceride, and diastolic blood pressure between the two groups. There was a significantly higher percentage of current smokers in the case group than in the control group, while there was no difference in the proportion of alcohol drinkers between the two groups. All cases had dyslipidemia with/without hypertension or diabetes mellitus and received statins.

\section{RBC membrane fatty acid composition}

Fatty acid composition of RBCs is presented in Table 2. No difference was observed regarding the RBC membrane contents of saturated fatty acids, PUFA, and total transfatty acids between the case-control groups, but monounsaturated fatty acids (MUFA) of RBC were significantly lower in cases than controls $(\mathrm{P}=0.011)$. Among saturated fatty acids, 18: 0 was lower in cases when compared to that found in controls and 24: 0 was higher. In regard to MUFA, 16: 1n-7t, 18: 1n-9, and 20: 1n-9 were significantly higher in cases than controls, whereas 16: 1n-7 was lower. Of RBC contents of PUFA, omega 6 fatty acids were significantly higher in cases than in controls $(\mathrm{P}<0.001)$, while omega 3 fatty acids were lower $(P=0.008)$. 
Table 2 Fatty acid composition of erythrocytes in subjects

\begin{tabular}{|c|c|c|c|}
\hline Fatty acids (weight \%) & Case $(n=24)$ & Control $(n=68)$ & $\mathrm{p}$ value ${ }^{*}$ \\
\hline Saturated fatty acids & $39.45 \pm 6.45$ & $40.12 \pm 4.90$ & 0.600 \\
\hline Myristic (14:0) & $0.77 \pm 0.41$ & $0.65 \pm 0.43$ & 0.227 \\
\hline Palmitic (16:0) & $24.31 \pm 3.44$ & $23.63 \pm 3.30$ & 0.390 \\
\hline Stearic (18:0) & $13.71 \pm 3.64$ & $15.34 \pm 2.79$ & 0.025 \\
\hline Lignoceric (24:0) & $0.67 \pm 0.47$ & $0.43 \pm 0.51$ & 0.048 \\
\hline Monounsaturated fatty acids & $17.09 \pm 1.91$ & $18.22 \pm 1.81$ & 0.011 \\
\hline trans-Palmitoleic (16:1n-7t) & $0.60 \pm 0.55$ & $0.36 \pm 0.42$ & 0.031 \\
\hline Palmitoleic (16:1n-7) & $1.11 \pm 0.48$ & $4.40 \pm 2.87$ & $<0.001$ \\
\hline trans-Oleic (18:1n-9t) & $0.84 \pm 0.41$ & $0.58 \pm 0.65$ & 0.071 \\
\hline Oleic $(18: 1 n-9)$ & $14.65 \pm 1.70$ & $12.96 \pm 2.43$ & 0.002 \\
\hline Eicosenoic (20:1n-9) & $0.79 \pm 0.50$ & $0.31 \pm 0.37$ & $<0.001$ \\
\hline Nervonic (24:1n-9) & $0.54 \pm 0.45$ & $0.36 \pm 0.88$ & 0.062 \\
\hline Polyunsaturated fatty acids & $41.35 \pm 7.36$ & $40.01 \pm 5.45$ & 0.351 \\
\hline Omega-6 & $29.81 \pm 5.01$ & $26.05 \pm 4.14$ & $<0.001$ \\
\hline trans-Linoleic (18:2n-6t) & $0.22 \pm 0.17$ & $0.57 \pm 0.51$ & $<0.001$ \\
\hline Linoleic (18:2n-6) & $14.38 \pm 4.59$ & $10.54 \pm 3.42$ & $<0.001$ \\
\hline Gamma-linolenic (18:3n-6) & $0.34 \pm 0.18$ & $0.27 \pm 0.56$ & 0.590 \\
\hline Eicosadienoic (20:2n-6) & $0.33 \pm 0.18$ & $0.41 \pm 0.48$ & 0.425 \\
\hline Dihomo-GLA (20:3n-6) & $1.72 \pm 0.46$ & $1.43 \pm 0.38$ & 0.003 \\
\hline Arachidonic (20:4n-6) & $11.25 \pm 3.07$ & $11.25 \pm 2.76$ & 0.998 \\
\hline Adrenic (22:4n-6) & $1.29 \pm 0.60$ & $1.53 \pm 0.61$ & 0.101 \\
\hline Docosapentaenoic (22:5n-6) & $0.51 \pm 0.30$ & $0.48 \pm 0.61$ & 0.753 \\
\hline Omega-3 & $11.54 \pm 3.58$ & $13.96 \pm 3.86$ & 0.008 \\
\hline Alpha-linolenic (18:3n-3) & $0.77 \pm 0.43$ & $0.60 \pm 0.66$ & 0.148 \\
\hline Eicosapentaenoic (20:5n-3) & $1.80 \pm 0.65$ & $2.53 \pm 0.96$ & $<0.001$ \\
\hline Docosapentaenoic (22:5n-3) & $1.93 \pm 0.73$ & $2.64 \pm 0.91$ & 0.001 \\
\hline Docosahexaenoic (22:6n-3) & $7.03 \pm 2.54$ & $8.61 \pm 2.57$ & 0.011 \\
\hline$n-6 / n-3$ & $2.79 \pm 0.83$ & $2.07 \pm 0.86$ & 0.001 \\
\hline Omega-3 index & $8.83 \pm 3.00$ & $11.13 \pm 3.08$ & 0.002 \\
\hline AA/EPA & $6.88 \pm 2.84$ & $5.07 \pm 2.20$ & 0.002 \\
\hline Total trans fatty acids & $1.51 \pm 0.68$ & $1.39 \pm 0.86$ & 0.527 \\
\hline
\end{tabular}

*By two sample $t$-test; Omega-3 index, docosahexaenoic acid + eicosapentaenoic acid \%; AA, arachidonic acid; EPA, eicosapentaenoic acid

In omega 6 fatty acids, only 18: $2 \mathrm{n}-6 \mathrm{t}$ was lower in cases than in controls, but 18: $2 n-6$ and 20: $3 n-6$ were higher. Cases had significantly lower RBC membrane contents of 20: 5n-3 (EPA), 22: 5n-3, and 22: 6n-3 (DHA) than did controls. The omega 6/omega 3 ratio and arachidonic acid/EPA ratio were significantly higher, but the omega-3 index was significantly lower in cases than in controls $(\mathrm{P}=$ 0.002).

\section{Correlation between acute STEMI, alcohol drinking,} smoking, BMI, and RBC membrane fatty acid composition The partial correlation coefficients between acute STEMI, alcohol drinking, smoking, BMI, and RBC membrane fatty acid composition are shown in Table 3. There was a negative correlation between acute STEMI and quartiles of the omega- 3 index $(\mathrm{r}=-0.585, \mathrm{P}<$ $0.001)$, BMI $(\mathrm{r}=-0.272, P=0.027)$, and MUFA $(\mathrm{r}=$ -0.299, $\mathrm{P}=0.015)$, while there was a borderline correlation between acute STEMI and fish consumption ( $\mathrm{r}=-0.242, \mathrm{P}=0.050)$ after adjusting for age and gender. However, acute STEMI correlated positively with smoking $(\mathrm{r}=0.366, \mathrm{P}=0.003)$ and the arachidonic acid/EPA ratio $(r=0.309, P=0.012)$ after adjusting for age and gender.

\section{Association of the omega-3 index, smoking, and odds for} being acute STEMI

Study subjects were categorized into four groups according to the omega-3 index. Multivariable adjusted regression analysis showed that the omega-3 index, age; the omega- 3 index was inversely associated with odds for being a case (OR 0.16 (95\% CI 0.03-1.14); $\mathrm{P}=$ 0.047 ), while smoking was positively associated with odds for being a case (OR 6.67 (95\% CI 1.77-25.23); P = $0.005)$ after adjusting for age, gender, BMI, alcohol drinking (Table 4). 
Table 3 Correlation between acute myocardial infarction, alcohol drinking, smoking, body mass index, and erythrocyte membrane fatty acid composition

\begin{tabular}{lcc}
\hline Variables & Acute $\mathbf{M l}$ & Omega-3 index \\
\hline Omega-3 index & $-0.585(<0.001)$ & $-0.121(0.333)$ \\
Alcohol drinking & $-0.004(0.973)$ & $-0.220(0.076)$ \\
Smoking & $\mathbf{0 . 3 6 6 ( 0 . 0 0 3 )}$ & $\mathbf{0 . 2 9 3 ( 0 . 0 1 7 )}$ \\
Body mass index & $-\mathbf{0 . 2 7 2 ( 0 . 0 2 7 )}$ & $\mathbf{0 . 2 8 4}(\mathbf{0 . 0 2 1})$ \\
Fish intake (servings/week) & $-\mathbf{0 . 2 4 2 ( 0 . 0 5 0 )}$ & $-\mathbf{0 . 4 2 4}(<\mathbf{0 . 0 0 1 )}$ \\
Total saturated fatty acids & $-0.049(0.693)$ & $-0.213(0.086)$ \\
Total monounsaturated fatty acids & $-\mathbf{0 . 2 9 9 ( 0 . 0 1 5 )}$ & $\mathbf{0 . 4 9 9 ( < 0 . 0 0 1 )}$ \\
Total polyunsaturated fatty acids & $0.080(0.503)$ & $-\mathbf{0 . 4 7 3 ( < 0 . 0 0 1 )}$ \\
Arachidonic acid/eicosapentaenoic acid ratio & $\mathbf{0 . 3 0 9 ( 0 . 0 1 2 )}$ & $-0.150(0.231)$ \\
Total trans fatty acids & $0.117(0.350)$ &
\end{tabular}

By partial correlation coefficient after adjustment for age and gender

Data are expressed as correlation coefficients ( $p$ value).

\section{Discussion}

Statins are well-known as the first-line drugs in the treatment of hyper-LDL cholesterolemia. Large-scale, prospective, randomized clinical trials have demonstrated that statins improve clinical cardiovascular outcomes and reduce mortality in both primary and secondary prevention [18]. However, in the real world, despite that clinical evidence, CHD including acute MI can occur even among dyslipidemia patients currently taking a statin with an LDL goal of less than $100 \mathrm{mg} / \mathrm{d}$ [23]. Therefore, combining omega-3 fatty acids with a statin induces further triglyceride reduction for patients with high triglyceride levels despite achieving target LDL cholesterol goals. However, little is known about further prevention of cardiovascular events in hypercholesterolemia patients without hypertriglyceridemia, especially with the addition of fish oil capsules to statin therapy. A study did report, however, that there was a further decrease in nonfatal coronary events or unstable angina in patients with hypercholesterolemia, especially in secondary prevention with the use of statins

Table 4 Multivariate analysis of the odds for being a case by quartiles of the Omega-3 Index

\begin{tabular}{|c|c|c|}
\hline \multirow[b]{2}{*}{ Variables } & \multicolumn{2}{|c|}{ Acute myocardial infarction } \\
\hline & Odds ratio ( $p$ value) & 95\% C.I. \\
\hline \multicolumn{3}{|l|}{ Omega-3 index (\%) } \\
\hline $1^{\text {st }}$ quartile $(<8.11)^{*}$ & $6.383(0.047)$ & $1.022-39.853$ \\
\hline $2^{\text {nd }}$ quartile (8.11-11.00) & $2.032(0.471)$ & $0.072-14.001$ \\
\hline $3^{\text {rd }}$ quartile (11.00-13.05) & $2.982(0.258)$ & $0.105-19.823$ \\
\hline $4^{\text {th }}$ quartile (> 13.05) & 1.000 & \\
\hline $\mathrm{Age}^{*}$ & $1.073(0.032)$ & $1.006-1.144$ \\
\hline Sex & $1.301(0.701)$ & $0.340-4.980$ \\
\hline Body mass index & $1.019(0.866)$ & $0.822-1.262$ \\
\hline Alcohol drinker & $1.466(0.568)$ & $0.378-5.894$ \\
\hline Current Smoker* & $6.673(0.005)$ & $1.765-25.229$ \\
\hline
\end{tabular}

${ }^{*} \mathrm{p}<0.05$, Test for trend across quartiles of Omega-3 index $(P=0.01)$. and EPA in combination therapy [24]. This was the first study to show an additional clinical benefit of long-term combination therapy with omega- 3 fatty acids and statins without serious adverse effects compared to statins only. However, this trial was not designed as a double-blind study. Also, unfortunately, the study did not show RBC fatty acid composition, investigated only EPA and not a DHA effect on coronary events, and revealed no additional benefit for acute MI.

Omega-3 fatty acids are known to cause improvement in lipid profiles without inducing serious hepatotoxicity or myopathy. Unlike previous studies, our study was specifically designed to evaluate the fatty acid composition of $\mathrm{RBC}$ and lifestyle factors such as alcohol consumption and smoking status in patients who developed acute STEMI, although they were taking statins and had achieved their LDL cholesterol control. The findings of this study can give us better understanding of the effectiveness and limitation of statin use for primary prevention of acute MI.

In the present study, the average omega- 3 index in healthy controls was about $11 \%$, which was similar to figures from previous studies in Koreans [14,25], and slightly higher than those reported by previous studies performed in Japan $[26,27]$. Recent studies have shown that the Japanese have an omega-3 index of $7-11 \%$, and the index tends to rise along with increasing age [26,27]. Our study did not show a correlation between omega-3 index and age.

Recently, two case-control studies were conducted to reveal the omega- 3 index of RBCs in patients with acute MI or metabolic syndrome in Korea. One study found that patients with acute MI have a lower omega-3 index (9.6\%) in comparison to healthy controls, and total trans-fatty acids are associated with an increased risk of MI. However, the result did not have adequate statistical power due to a wide confidence interval of the odds ratio of MI risk estimate. The other study reported that 
patients with (11.8\%) and without metabolic syndrome (12.4\%) have similar omega-3 indices. In the present study, we found that patients who experienced acute STEMI, even though they were taking statins and had achieved their LDL cholesterol control goal, had an omega-3 index of $8.8 \%$, which was significantly lower than that of controls (11.1\%), while no difference in total trans-fatty acids was observed between the two groups. Previous studies have provided inconsistent data on whether trans fat intakes are associated with MI risk according to levels of intake [28-30]. On the other hand, the present study found that omega- 6 fatty acids such as linoleic acid were increased in patients with acute STEMI compared to controls, which was in accordance with a previous study [31]. Linoleic acid was frequently inversely associated with risk for coronary heart disease events in Western people [32]. However, the present study found that omega- 6 fatty acids such as linoleic acid were increased in patients with acute STEMI compared to controls. Previous studies from Korea and Japan showed that there were no differences of linoleic acid level between MI cases and controls [25,33]. Although it is not yet known why, possible mechanisms underlying the ethnic difference in linoleic acid concentrations include differences in fat distribution, fatty acid interactions with genetic polymorphisms, and diet. Replication studies with larger sample size would be needed to confirm our study results.

The American Heart Association recommends that healthy people should eat two servings of a variety of (preferably oily) fish per week [11]. The present study showed an association between fish consumption and the omega-3 index $(r=0.269, P=0.026)$, although two sample $t$-test revealed only a borderline difference of average fish consumption (servings/week) between cases and controls ( 2.1 vs. $3.1, \mathrm{P}=0.059$ ), although the consumption was greater than that in most Western populations. A Japanese study also showed that a very high level of fish intake was significantly associated with a lower risk of nonfatal CHD [17].

Harris et al. [13] suggest the omega-3 index (EPA + DHA as a percent of total fatty acid in RBC membranes) as a novel risk factor for CHD. The studies have shown that a higher omega-3 index than a value of $8-10 \%$ is desirable for its cardio-protective benefits, because of the relationship between a lower average omega- 3 index and the development of CHD in Caucasians [13,22]. Interestingly, Korean patients with acute STEMI already had an omega-3 index of $8 \%$, which is a cardio-protective goal for Western people. Therefore, we suggest that a higher cut-off point of omega-3 index than $8-10 \%$ for preventing CHD is needed, and an omega- 3 index of $11 \%$ or above could be appropriate in Korea. This discrepancy between Korean and Western people could be explained in large part by the fact that most Korean people have higher fish consumption than people of Western populations. Korean consumes fish species such as mackerel, salmon which are very high in omega-3 [34]. In addition, there may be ethnic differences in the threshold of omega-3 index and underlying mechanism of development of CHD. Using multivariable adjusted regression analysis, our study showed that lower omega-3 index was independently associated with acute STEMI after adjusting for age, sex, and BMI. Subjects in the lowest quartile of omega-3 index was positively associated with almost six times odds for being a case, even after adjusting for all confounding variables, than the highest quartile subgroup (Table 4). That finding is concordant with previous casecontrol studies about EPA and DHA levels that found significantly lower EPA and DHA in patients with acute coronary syndrome compared to healthy control people $[29,35]$. A case-control follow-up study also provides evidence of a plasma concentration of EPA and DPA associated with a lower incidence of nonfatal MI among American women [30], despite their current lower fish intake than that of typical Korean and Japanese people.

Cigarette smoking is an important risk factor for cerebro-cardiovascular diseases. Epidemiologic, clinical, and experimental data have revealed that smoking is prothrombotic, atherogenic, and causative factors in the development of CHD [36]. In the present study, we also found that smoking was a major associated factor for acute STEMI development in patients taking statins who had achieved their goal LDL cholesterol. Our results were consistent with a previous study using Japanese data that revealed an association of cigarette smoking with CVD mortality in patients, despite their lower total cholesterol level [37]. The relationship between omega-3 index and smoking status is a controversial topic [38]. In additional analysis, we found no difference in the omega-3 index between smokers and non-smokers within each group (data not shown).

There is no single cause for CHD. CVD is caused by a constellation of risk factors, including environmental factors, such as sedentary lifestyle, genetic predisposition, advancing age, smoking, an atherogenic diet, and underlying conditions or diseases including insulin resistance or type 2 diabetes, hypertension, and dyslipidemia. In this view, interestingly, the present study showed that smoking, a lower omega-3 index, and advancing age together among those factors may work together to cause acute STEMI despite statin-induced low LDL cholesterolemia. Another Korean study also reported that, compared to survivors, non-survivors of acute MI had a lower level of total cholesterol, triglycerides, and LDL-cholesterol, while the opposite was observed with plasma omega-3 fatty acids [39]. However, the researchers did not explain why non-survivors from acute MI had lower lipid levels 
than survivors. It is obvious, however, that certain cardiovascular events still occur even among patients reaching target levels of LDL-cholesterol $[30,35]$. Therefore, relative to controls, STEMI cases are more likely to be smokers and to have a lower omega-3 index, even though the LDL cholesterol target is achieved with a statin in the cases with dyslipidemia. Patients should be asked to quit smoking and eat more fish or take omega-3 supplements as well as reach their LDL-cholesterol target on a statin.

Our study is limited by its cross-sectional study design. Further investigation with a cohort study is warranted. Another limitation is the wide confidence interval due to a relatively small sample size. Koreans usually have a fish intake that is several times higher than Western people, which limits the generalizability of those results to other population groups.

In summary, subjects with acute STEMI had a lower omega-3 index and higher smoking rate after adjusting for age, gender, and BMI than controls, although they are currently receiving a statin with an LDL goal of less than $100 \mathrm{mg} / \mathrm{d}$ and normal triglyceride levels. Nevertheless, acute STEMI patients have an omega- 3 index of $8.8 \%$ that is higher than that of people of Western populations. This means that the cut-off point for the omega-3 index for preventing CVD might be tailored for the Korean population. Thus, at present, we suggest a suitable level of an omega- 3 index of $11 \%$ or above for prevention of coronary artery disease in the Korean population. However, replication studies with a larger sample size are needed to confirm our first stage study results for current practice in the field.

\section{Funding/Support}

This work was supported by a Medical Research Institute Grant (2007-9) from Pusan National University, Busan, Korea

\section{Author details \\ 'Department of Family Medicine, Pusan National University Hospital, Busan, South Korea. ${ }^{2}$ Family Medicine Clinic and Research Institute of Convergence of Biomedical Science and Technology, Pusan National University Yangsan Hospital, Yangsan, South Korea. ${ }^{3}$ Division of Cardiology, Department of Internal Medicine, Pusan National University Hospital, Busan, South Korea. ${ }^{4}$ Medical Education Unit and Medical Research Institute, Pusan National University School of Medicine, Yangsan, South Korea. ${ }^{5}$ Department of Food \& Nutrition, Hanyang University, Seoul, South Korea. ${ }^{6}$ Family Medicine Clinic, Pusan National University Yangsan Hospital, Beomeo-ri, Mulgeum-eup, Yangsan, Gyeongsangnam-do 626-770, South Korea.}

\section{Authors' contributions}

SYL had full access to all of the data in the study and takes responsibility for the integrity of the data and the accuracy of the data analysis. YJK, JGL and SYL participated in the study concept and design. YJK, HCL and YHC participated in acquisition of data. YJK, SYL and YSP participated in analysis and interpretation of data. SYL and JGL participated in drafting of the manuscript. SJI, MJB and EJC participated in critical revision of the manuscript for important intellectual content. SYL and DWJ carried out statistical analysis. JGL, YHC, MJB and EJC participated in administrative, technical, or material support. All authors read and approved the final manuscript.

\section{Competing interests}

All authors have completed and submitted the ICMJE Form for Disclosure of Potential Conflicts of Interest.

Received: 2 January 2012 Accepted: 27 March 2012

Published: 27 March 2012

References

1. Callow AD: Cardiovascular disease 2005-the global picture. Vascul Pharmacol 2006, 45(5):302-307.

2. Prasad DS, Kabir Z, Dash AK, Das BC: Smoking and cardiovascular health: a review of the epidemiology, pathogenesis, prevention and control of tobacco. Indian J Med Sci 2009, 63(11):520-533.

3. Franco $M$, Cooper RS, Bilal U, Fuster V: Challenges and opportunities for cardiovascular disease prevention. Am J Med 2011, 124(2):95-102.

4. Stampfer MJ, Hu FB, Manson JE, Rimm EB, Willett WC: Primary prevention of coronary heart disease in women through diet and life style. $N$ Engl Med 2000, 343:16-22

5. Brien SE, Ronksley PE, Turner BJ, Mukamal KJ, Ghali WA: Effect of alcohol consumption on biological markers associated with risk of coronary heart disease: systematic review and meta-analysis of interventional studies. BMJ 2011, 342:d636.

6. von Schacky C: Omega-3 fatty acids and cardiovascular disease. Curr Opin Clin Nutr Metab Care 2007, 10(2):129-135.

7. Deutch B, Dyerberg J, Pedersen HS, Aschlund E, Hansen JC: Traditional and modern Greenlandic food-dietary composition, nutrients and contaminants. Sci Total Environ 2007, 384(1-3):106-119.

8. Wang $C$, Harris WS, Chung M, Lichtenstein AH, Balk EM, Kupelnick B, Jordan HS, Lau J: n-3 Fatty acids from fish or fish-oil supplements, but not alpha-linolenic acid, benefit cardiovascular disease outcomes in primary- and secondary-prevention studies: a systematic review. Am J Clin Nutr 2006, 84(1):5-17.

9. Galli C, Calder PC: Effects of fat and fatty acid intake on inflammatory and immune responses: a critical review. Ann Nutr Metab 2009, 55(13):123-139.

10. Robinson JG, Stone NJ: Antiatherosclerotic and antithrombotic effects of omega-3 fatty acids. Am J Cardiol 2006, 98(4A):39i-49i.

11. Harris WS, Bulchandani D: Why do omega-3 fatty acids lower serum triglycerides? Curr Opin Lipidol 2006, 17(4):387-393.

12. Mozaffarian D: Fish, n-3 fatty acids, and cardiovascular haemodynamics. $J$ Cardiovasc Med 2007, 8(Suppl 1):S23-S26.

13. Harris WS: The omega-3 index: clinical utility for therapeutic intervention. Curr Cardiol Rep 2010, 12(6):503-508.

14. Lee $E$, Lee $S$, Park $Y: n-3$ Polyunsaturated fatty acids and trans fatty acids in patients with the metabolic syndrome: a case-control study in Korea. Br J Nutr 2008, 100(3):609-614.

15. Itomura M, Fujioka S, Hamazaki K, Kobayashi K, Nagasawa T, Sawazaki S, Kirihara Y, Hamazaki T: Factors influencing EPA + DHA levels in red blood cells in Japan. In Vivo 2008, 22(1):131-135.

16. Lee K: Cardiovascular risk prevalence, awareness, treatment, and control from 1998 to 2007 in Koreans. Asia Pac J Clin Nutr 2010, 19(2):261-265.

17. Iso H, Kobayashi M, Ishihara J, Sasaki S, Okada K, Kita Y, Kokubo Y, Tsugane S, JPHC Study Group: Intake of fish and n3 fatty acids and risk of coronary heart disease among Japanese: the Japan Public Health Center-Based (JPHC) Study Cohort I. Circulation 2006, 113(2):195-202.

18. Taylor F, Ward K, Moore TH, Burke M, Davey Smith G, Casas JP, Ebrahim S: Statins for the primary prevention of cardiovascular disease. Cochrane Database Syst Rev 2011, , 1: CD004816.

19. Davidson MH, Stein EA, Bays HE, Maki KC, Doyle RT, Shalwitz RA, Ballantyne CM, Ginsberg HN, COMBination of prescription Omega-3 with Simvastatin (COMBOS) Investigators: Efficacy and tolerability of adding prescription omega-3 fatty acids $4 \mathrm{~g} / \mathrm{d}$ to simvastatin $40 \mathrm{mg} / \mathrm{d}$ in hypertriglyceridemic patients: an 8-week randomized, double-blind, placebo-controlled study. Clin Therapeutics 2007, 29(7):1354-1367.

20. Maki KC, McKenney JM, Reeves MS, Lubin BC, Dicklin MR: Effects of adding prescription omega-3 acid ethyl esters to simvastatin ( $20 \mathrm{mg} /$ day) on lipids and lipoprotein particles in men and women with mixed dyslipidemia. Am J Cardiol 2008, 102(4):429-433. 
21. Davidson MH, Maki KC, Bays H, Carter R, Ballantyne CM: Effects of prescription omega-3-acid ethyl esters on lipoprotein particle concentrations, apolipoproteins Al and CIII, and lipoprotein-associated phospholipase $A(2)$ mass in statin-treated subjects with hypertriglyceridemia. J Clin Lipidol 2009, 3(5):332-340.

22. Harris WS, Von Schacky C: The Omega-3 Index: a new risk factor for death from coronary heart disease? Prev Med 2004, 39(1):212-220.

23. Big gaps in coronary heart disease prevention. Statins are not enough in dyslipidemia. MMW Fortschr Med 2009, 151(46):48.

24. Yokoyama M, Origasa H, Matsuzaki M, Matsuzawa Y, Saito Y, Ishikawa $Y$, Oikawa S, Sasaki J, Hishida H, Itakura H, Kita T, Kitabatake A, Nakaya N, Sakata T, Shimada K, Shirato K, Japan EPA lipid intervention study (JELIS) Investigators: Effects of eicosapentaenoic acid on major coronary events in hypercholesterolaemic patients (JELIS): a randomized open-label, blinded endpoint analysis. Lancet 2007, 369(9567):1090-1098.

25. Park Y, Lim J, Lee J, Kim SG: Erythrocyte fatty acid profiles can predict acute non-fatal myocardial infarction. Br J Nutr 2009, 102(9):1355-1361.

26. Yanagisawa N, Shimada K, Miyazaki T, Kume A, Kitamura Y, Ichikawa R, Ohmura H, Kiyanagi T, Hiki M, Fukao K, Sumiyoshi K, Hirose K, Matsumori R, Takizawa H, Fujii K, Mokuno H, Inoue N, Daida H: Polyunsaturated fatty acid levels of serum and red blood cells in apparently healthy Japanese subjects living in an urban area. J Atheroscler Thromb 2010, 17(3):285-294.

27. Kawabata T, Hirota S, Hirayama T, Adachi N, Hagiwara C, Iwama N, Kamachi K, Araki E, Kawashima H, Kiso Y: Age-related changes of dietary intake and blood eicosapentaenoic acid, docosahexaenoic acid, and arachidonic acid levels in Japanese men and women. Prostaglandins Leukot Essent Fatty Acids 2011, 84(5-6):131-137.

28. Oh K, Hu FB, Manson JE, Stampfer MJ, Willett WC: Dietary fat intake and risk of coronary heart disease in women: 20 years of follow-up of the nurses' health study. Am J Epidemiol 2005, 161:672-679.

29. Harris WS, Reid KJ, Sands SA, Spertus JA: Blood omega-3 and trans fatty acids in middle-aged acute coronary syndrome patients. Am J Cardiol 2007, 99(2):154-158.

30. Sun Q, Ma J, Campos H, Rexrode KM, Albert CM, Mozaffarian D, Hu FB: Blood concentrations of individual long-chain $n-3$ fatty acids and risk or nonfatal myocardial infarction. Am J Clin Nutr 2008, 88(1):216-233.

31. Block RC, Harris WS, Reid KJ, Spertus JA: Omega- 6 and trans fatty acids in blood cell membranes: a risk factor for acute coronary syndromes? Am Heart J 2008, 156(6):1117-1123.

32. Harris WS, Poston WC, Haddock CK: Tissue n-3 and n-6 fatty acids and risk for coronary heart disease events. Atherosclerosis 2007, 193(1):1-10.

33. Oda E, Hatada K, Katoh K, Kodama M, Nakamura Y, Aizawa Y: A casecontrol pilot study on $\mathrm{n}-3$ polyunsaturated fatty acid as a negative risk factor for myocardial infarction. Int Heart J 2005, 46(4):583-591.

34. Tsuchiya A, Hardy J, Burbacher TM, Faustman EM, Mariën K: Fish intake guidelines: incorporating $n-3$ fatty acid intake and contaminant exposure in the Korean and Japanese communities. Am J Clin Nutr 2008, 87(6):1867-1875

35. Block RC, Harris WS, Reid KJ, Sands SA, Spertus JA: EPA and DHA in blood cell membranes from acute coronary syndrome patients and controls. Atherosclerosis 2008, 197:821-828.

36. Reid RD, Mullen KA, Pipe AL: Systematic approaches to smoking cessation in the cardiac setting. Curr Opin Cardiol 2011, 26(5):443-448.

37. Hozawa A, Okamura T, Kadowaki T, Murakami Y, Nakamura K, Hayakawa T, Kita Y, Nakamura Y, Okayama A, Hirotsugu Ueshima for NIPPON DATA80 Research group: Is weak association between cigarette smoking and cardiovascular disease mortality observed in Japan explained by low total cholesterol? NIPPON DATA80. Int J Epidemiol 2007, 36(5):1060-1067.

38. Block RC, Harris WS, Pottala JV: Determinants of Blood Cell Omega-3 Fatty Acid Content. Open Biomark J 2008, 1:1-6.

39. Lee SH, Shin MJ, Kim JS, Ko YG, Kang SM, Choi D, Jang Y, Chung N, Shim WH, Cho SY, Manabe I, Ha JW: Blood eicosapentaenoic acid and docosahexaenoic acid as predictors of all-cause mortality in patients with acute myocardial infarction-data from Infarction Prognosis Study (IPS) Registry. Circ J 2009, 73(12):2250-2257.

doi:10.1186/1476-511X-11-43

Cite this article as: Kim et al:: Omega-3 index and smoking in patients with acute ST-elevation myocardial infarction taking statins: a casecontrol study in Korea. Lipids in Health and Disease 2012 11:43.

\section{Submit your next manuscript to BioMed Central and take full advantage of:}

- Convenient online submission

- Thorough peer review

- No space constraints or color figure charges

- Immediate publication on acceptance

- Inclusion in PubMed, CAS, Scopus and Google Scholar

- Research which is freely available for redistribution 